\title{
Wearable Sensorimotor Enhancer for Fingertip based on Stochastic Resonance Effect
}

\author{
Yuichi Kurita, Member, IEEE, Minoru Shinohara, Jun Ueda, Member, IEEE
}

\begin{abstract}
This paper reports on the results of an experiment involving a wearable sensorimotor enhancer intended to improve tactile sensitivity in human fingertips. As briefly exposing tactile receptors to sub-sensory vibration is known to enhance tactile sensitivity thanks to a phenomenon called stochastic resonance (SR) in the somatosensory system, applying white-noise vibration to a fingertip is expected to improve the sense of touch and associated motor skills. Against such a background, a prototype of a wearable device called a sensorimotor enhancer is proposed here. The device is attached to the radial side of the fingertip and stimulates tactile receptors by applying vibration from a lead zirconate titanate (PZT) piezoelectric stack actuator. This design keeps the palmar region free, thereby helping to maintain the wearer's manipulative ability. Sensory and motor tests involving 11 human subjects were conducted to determine the efficacy of the device, and the results confirmed its usefulness.
\end{abstract}

Index Terms-Stochastic Resonance, Wearable Device, Fingertip, Tactile Sensitivity, Motor Performance.

\section{INTRODUCTION}

Stochastic resonance (SR) is known to improve the sensitivity of a non-linear system in the presence of very weak noise [1], [2], [3]. SR has been observed in the mechanoreceptors of animals such as crayfish, toads and rats [4], [5], [6], and it is known that the sensitivity of somatosensory receptors can be improved by short-term exposure to sub-sensory vibration. Some studies have also reported that SR improves visual[9], aural [10] and haptic [11] ability. The SR effect in tactile sensitivity has also been confirmed in feet [12], [13], hands and fingers [14], [15], and improved tactile sensitivity is known to influence the sensorimotor functions of humans[7], [8]. It has further been reported that $\mathrm{SR}$ improves balance control in humans [16], [17]. .

However, the effects of SR on dexterity and finger force control remain unclear. In this context, the authors hypothesized that improved tactile sensory capability in fingertips would help to enhance motor performance in finger-based

Y. Kurita is with the Graduate School of Engineering, Hiroshima University, 1-4-1 Kagamiyama, Higashi-Hiroshima, Hiroshima 739-8527 Japan

E-mail: kurita@bsys.hiroshima-u.ac.jp

M. Shinohara is with the School of Applied Physiology, Georgia Institute of Technology, 555 14th Street NW, Atlanta, GA 30332 USA, and with the Rehabilitation R\&D Center of Excellence, Atlanta VA Medical Center, 670 Clairmont Road, Decatur, GA 30033 USA

J. Ueda is with the George W. Woodruff School of Mechanical Engineering, Georgia Institute of Technology, 771 Ferst Drive, Atlanta, GA 30332 USA

Yuichi Kurita expresses his gratitude to Japan Science and Technology Agency (JST) - National Science Foundation (NSF) Strategic International Cooperative Program. Minoru Shinohara is supported in part by VA MERIT Pilot Award (1 I01 RX000421-01). tasks. For example, a fingertip-wearable device that improves motor performance could assist workers engaged in tasks requiring high-precision manual dexterity. Although a variety of such devices have been developed in the past (including haptic interfaces [18], [19], sensing devices [20], [21], a vibration sensitivity improvement device [22] and devices for sensory visualization [23]), to the authors' knowledge, no wearable attachable device that explicitly leverages the effects of SR has yet been developed. As humans generally use the palmar surfaces of the fingertips to identify materials and handle objects, the palmar surface should be left free to maintain the manipulation ability of fingers.

This paper describes a wearable device called a sensorimotor enhancer, which is expected to improve the tactile sensitivity of fingertips. The proposed device has two important qualities. The first is its utilization of a piezoelectric stack actuator featuring high-frequency vibration generation capacity in a compact body. The second is its design, which ensures dexterity by leaving the palmar region of the fingertips free.

The concept of the sensorimotor enhancer and related preliminary results were reported in [24]. However, this paper adds more detail on the device, presents the results of experimentation with a greater number of subjects with higher statistical significance, and includes further discussion. The influence of the proposed sensorimotor enhancer on tactile sensitivity and motor performance was investigated in sensory and motor tests. To validate improvements in passive and active tactile sensitivity, three tests were carried out: a two-point discrimination test, a one-point touch test, and a texture discrimination test using pieces of sandpaper. In addition, a minimal-force grasping test was conducted to examine motor performance improvement. The results showed that all the tests produced statistically significant data that supported the validity of the sensorimotor enhancer concept.

\section{Methods}

\section{A. Sensorimotor enhancer}

Fig. 1 shows a glove-type wearable sensorimotor enhancer prototype. A compact lead zirconate titanate (PZT) piezoelectric stack actuator and a battery for the actuator are attached to or embedded inside the glove. The actuator is used to generate white-noise vibration that is transmitted to tactile receptors around the finger pulp. Fig. 2 shows the simple setup of the sensorimotor enhancer.

The device was fabricated with a latex finger cap and a piezoelectric actuator. Its palmar side is cut open so 


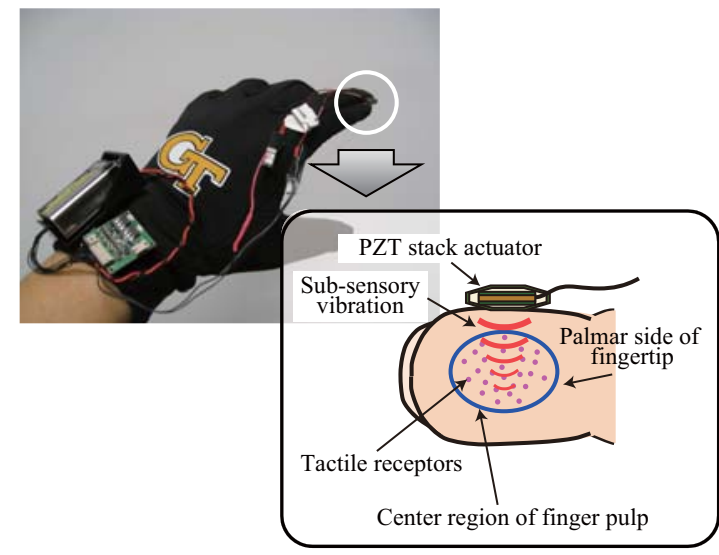

Fig. 1. Glove-type sensorimotor enhancer

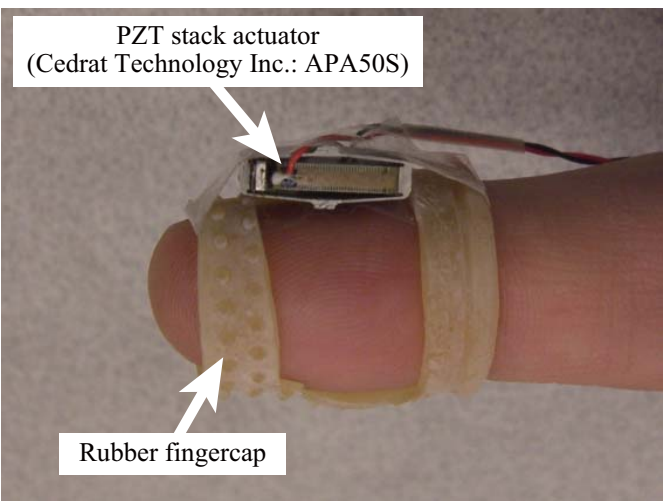

Fig. 2. Fingercap-type wearable device with a vibration generator

that the user can touch an object's surface directly. In this study, all the experiments were performed with this fingercap-type sensorimotor enhancer. LabView software was used to generate reference commands to the linear amplifier of the piezoelectric actuator. The range of the vibration frequency applied to the fingertip was determined in consideration of the frequency response characteristics of the tactile mechanoreceptors, which are known to have either rapidly adapting (RA) or slowly adapting (SA) characteristics. There are two subclasses of RA and SA receptors: RA I (Meissner corpuscles) and RA II (Pacinian corpuscles), and SA I (Merkel cells) and SA II (Ruffini endings). Pacinian corpuscles (PCs) are extremely sensitive at the optimal vibration frequency of $250[\mathrm{~Hz}]$ in young adults. The other three receptors are less sensitive, mainly due to their inability to exhibit spatial summation [25], [26]. Considering the sensitivity of the tactile receptors, vibration with a cutoff frequency of $300[\mathrm{~Hz}]$ was adopted in this study. Gaussian white noise was generated and treated with a second-order low-pass Butterworth filter applied by the LabView program. The signal amplitudes were individually determined based on the vibration perception capability of each subject.

\section{B. Experiments}

Four tests (two passive sensory tests, one active sensory test and one motor test) were conducted with 11 healthy

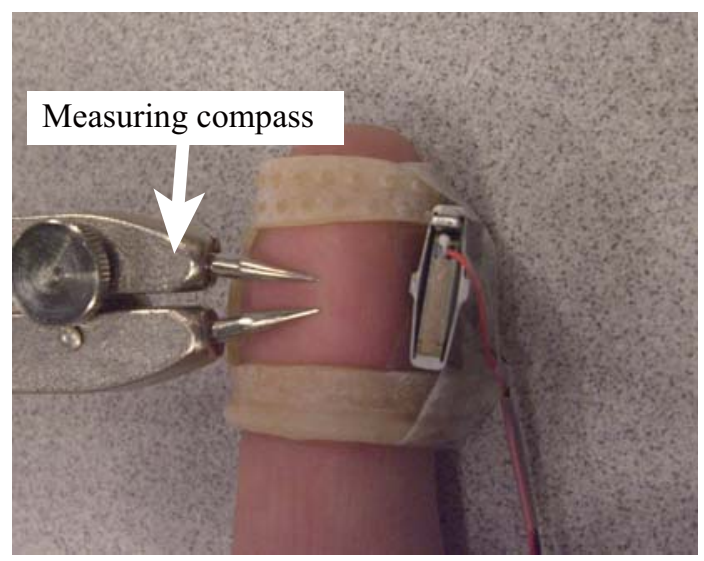

Fig. 3. Two-point discrimination test: a measuring compass was pressed against the palmar side of the fingertip.

subjects ( 8 males; 3 females) with ages ranging from 24 to 38 (mean: $30.5 \pm \mathrm{SD} 4.92$ ). The sensorimotor enhancer was attached to the subject's non-dominant index finger, and the hand was placed on a desk with the palmar surface upward. The subjects were instructed to relax their torso and dominant hand in order to minimize unwanted movement.

The amplitude threshold of vibration perception for each subject was determined prior to the experiment. A PZT actuator was used to apply white noise to the subject's fingertip from the radial surface. The subject was asked to report the perception of vibration at the contact point with the actuator when the signal amplitude changed. The maximum amplitude that the subject could not feel (i.e., the maximum sub-sensory amplitude) was recorded using a limit-based method. Three series of ascending and descending intensities (i.e., a total of six) were alternately applied, and the average was taken as the perception threshold. The vibration amplitude interval was $0.2[\mu \mathrm{m}]$. In the subsequent experiments, no-vibration and five different amplitude conditions (with 50, 75, 100, 125 and 150 [\%] of the perception threshold, denoted as $0.5 \mathrm{~T}, 0.75 \mathrm{~T}, 1.0 \mathrm{~T}$, $1.25 \mathrm{~T}$ and $1.5 \mathrm{~T}$, respectively) were applied in random order. A sole experimenter controlled the vibration amplitude applied in each task and recorded the results. Subjects were not informed of the vibration amplitude. The experimental protocol was reviewed and approved by the Georgia Institute of Technology Institutional Review Board, and all subjects gave informed consent before participating.

1) Two-point discrimination test: The subjects were instructed to place the hand with the sensorimotor enhancer attached on a table and close their eyes. The experimenter gently pressed two sharp points of a measuring compass against the palmar surface of each subject's fingertip as shown in Fig. 3. The subjects were asked to report when they felt definite contact from the two points. Two series of ascending and descending distances between the points (i.e., a total of four) were tested for each vibration condition, and the average was recorded as the result of each trial. The distance interval was $0.5[\mathrm{~mm}]$. The measured 


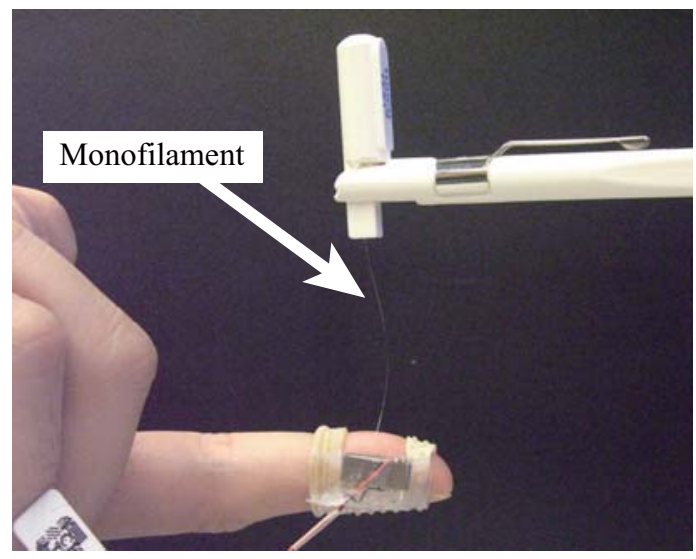

Fig. 4. One-point touch test: a monofilament was pressed against the palmar side of the fingertip.

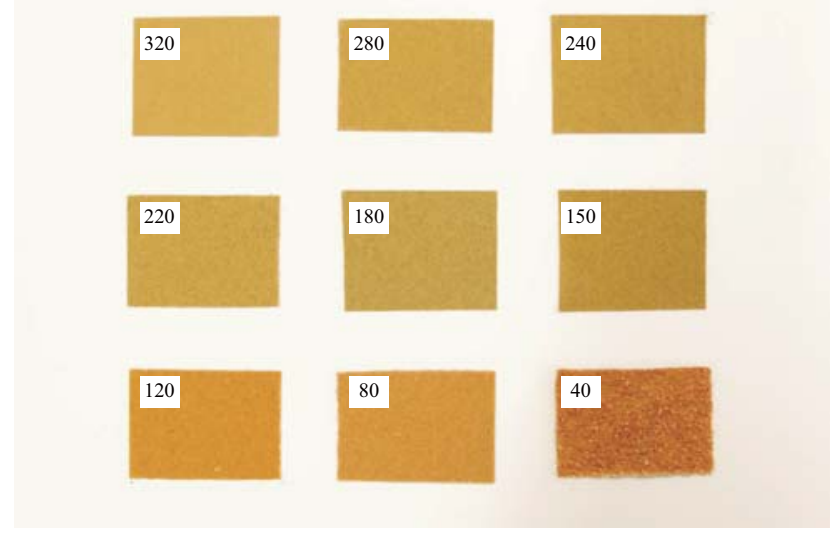

Fig. 5. Sandpapers used in the texture discrimination test: numbers indicate US CAMI grit sizes.

distance in the controlled case was normalized against the mean distance measured in the no-vibration case. Each trial lasted 90 seconds, and 30-second rest periods were provided between trials. Each subject underwent two trials for each vibration condition.

2) One-point touch test: The subjects were instructed to place the hand with the sensorimotor enhancer attached on a table and close their eyes. The experimenter pressed a monofilament against the palmar surface of each subject's fingertip until buckling occurred, held it for approximately 1.0 [sec] and then removed it. The subjects were asked to report when they felt contact from the filament. Two series of ascending and descending loads (i.e., a total of four) were tested for each vibration condition, and the average was recorded as the result of each trial. A total of five SemmesWeinstein monofilaments (Touch-Test Sensory Evaluator; $0.008,0.02,0.04,0.07$ and $0.1[\mathrm{~g}]$ ) were used as stimuli. The measured load in the controlled case was normalized against the mean load measured in the no-vibration case. Each trial lasted 60 seconds, and 30-second rest periods were provided between trials. Each subject underwent two trials for each vibration condition. Fig. 4 shows an overview of the experiment.

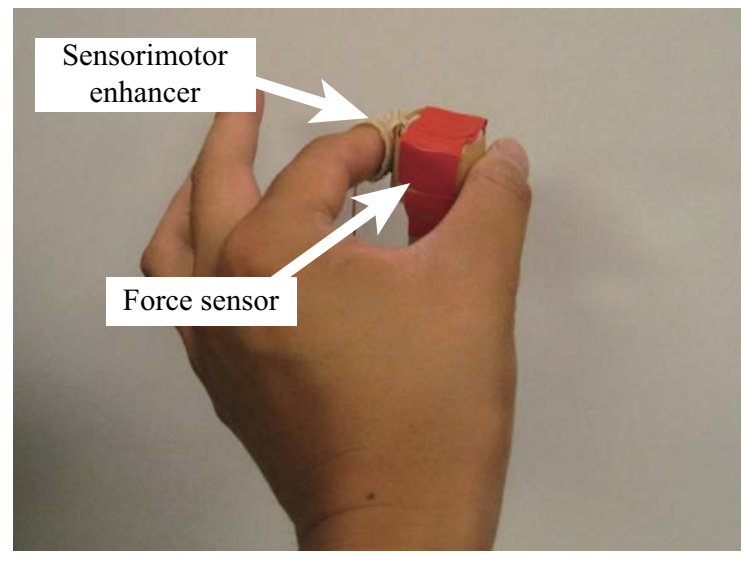

Fig. 6. Minimal force grasping test: the grasping force applied was measured by a force sensor equipped inside the object.

3) Active sensory test: Texture discrimination: In this experiment, pieces of sandpaper with CAMI grit sizes of $\# 40$, \#80, \#120, \#150, \#180, \#220, \#240, \#280 and \#320 were used as shown in Fig. 5. All the pieces were glued to one side of a plastic board provided to the subjects, who were not allowed to see the sandpaper but were permitted to touch and feel it. Attached to the other side of the board was a test piece of sandpaper with a grit size matching one of the nine sandpaper types. As the difficulty of identifying the sandpaper type depends on the grit size, the types were divided into four groups with grit sizes of 320 and 280; 240 and 220; 180 and 150; and 120, 80 and 40. One type from each group was randomly picked as the test sandpaper. In total, four sandpaper types (each corresponding to a different group) were tested in random order, and the average was recorded as one trial. The subjects were asked to touch the nine pieces of sandpaper and select the one they judged to have the same texture as the piece on the other side. Each subject underwent one trial for each vibration condition, and 30-second rest periods were provided between trials.

4) Motor skill test: Minimal force grasping: The sensorimotor enhancer was attached to the subject's index finger. As shown in Fig. 6, the subject was asked to pinch and hold an object with a weight of 140 [g] for 3 seconds with as little force as possible without allowing it to slip. The object was covered with plain printer paper and was equipped with a force sensor to record the grasping force between the index finger and thumb. Subjects who dropped the object were allowed to repeat the trial without penalty. The force measured in the controlled case was normalized against the mean force measured in the no-vibration case. Each subject underwent two trials for each vibration condition, and 30second rest periods were provided between trials.

\section{RESUlts AND Discussion}

\section{A. Experimental results}

1) Two-point discrimination test: Fig. 7 shows the results of the experiments. The horizontal axis indicates the 


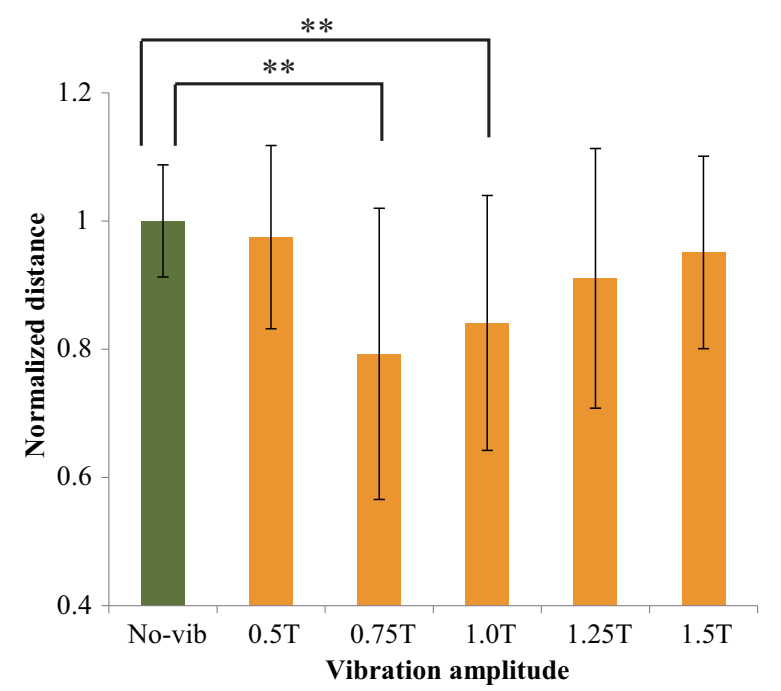

Fig. 7. Results of two-point test: discriminable distances in all five controlled cases were smaller than that in the no-vibration case. Statistically significant differences were observed for $0.75 \mathrm{~T}$ and $1.0 \mathrm{~T}$ cases $(* *$ means $p<0.01)$.

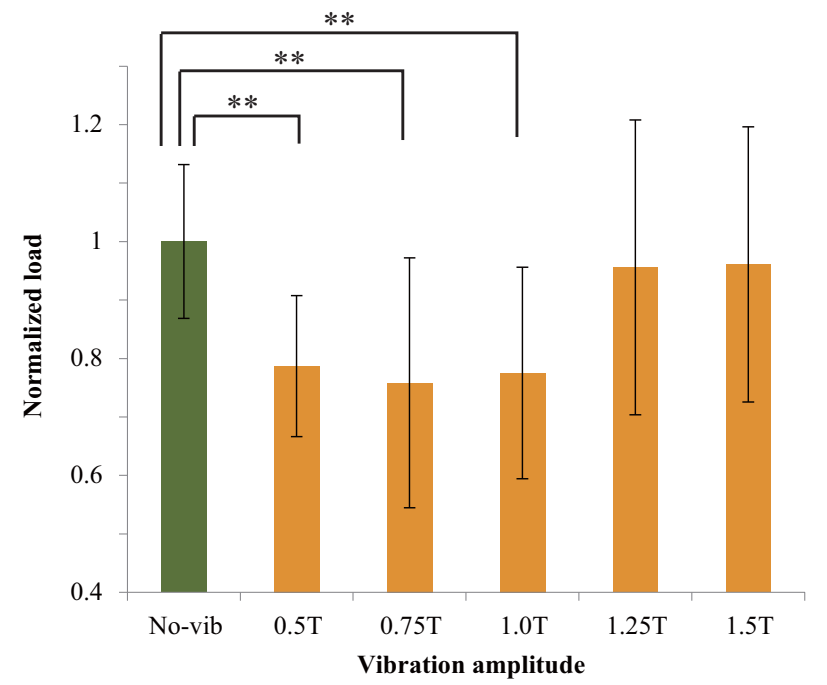

Fig. 8. Results of one-point test: perceivable forces in all five controlled cases were smaller than that in the no-vibration case. Statistically significant differences were observed for $0.5 \mathrm{~T}, 0.75 \mathrm{~T}$, and $1.0 \mathrm{~T}(* *$ means $p<0.01)$.

vibration amplitude, and the vertical axis indicates the normalized distance. The results show that the mean distances in all five controlled cases were smaller than that in the no-vibration case. One-way analysis of variance (ANOVA) showed a significant main effect from the vibration amplitude $\left(F(5,60)=3.16, p=0.013, \eta_{p}{ }^{2}=0.208\right)$. The results of post-hoc Dunnett testing also revealed significant differences against the no-vibration case for the $0.75 \mathrm{~T}$ $(p<0.01)$ and 1.0T $(p<0.01)$ cases.

2) One-point touch test: Fig. 8 shows the results of the experiment. The horizontal axis indicates the vibration amplitude, and the vertical axis indicates the normalized load. Smaller mean forces equate to better tactile sensitivity. The results show that the forces in all five controlled cases were smaller than that in the non-vibration case. One-way

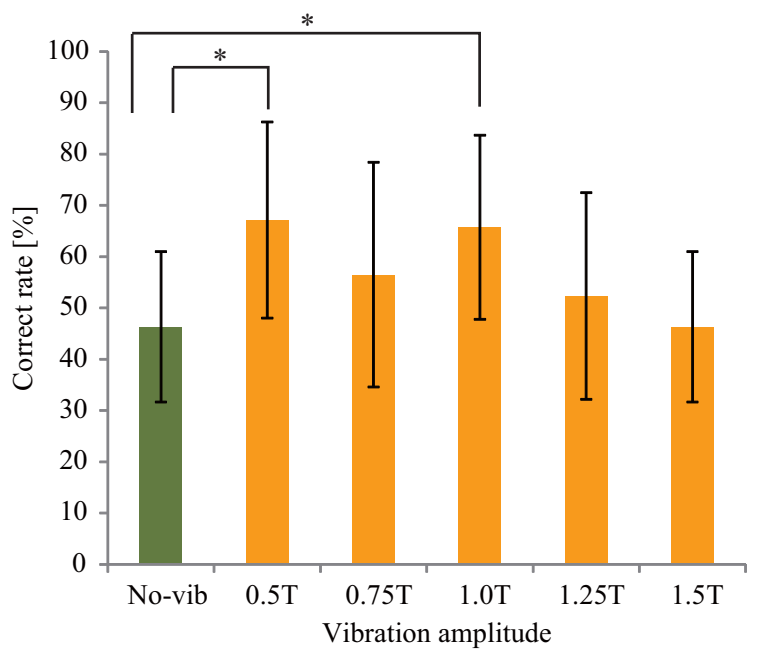

Fig. 9. Results of texture discrimination test: mean correct ratios in all five controlled cases were higher than that in the no-vibration case (0T). Statistically significant differences were observed for $0.5 \mathrm{~T}$ and $1.0 \mathrm{~T}$ cases (* means $p<0.05$ ).

ANOVA showed a significant main effect from the vibration amplitude $\left(F(5,60)=3.12, p=0.014, \eta_{p}{ }^{2}=0.206\right)$. The results of post-hoc Dunnett testing also revealed significant differences against the non-vibration case for the $0.5 \mathrm{~T}$ $(p<0.01), 0.75 \mathrm{~T}(p<0.01)$ and 1.0T $(p<0.01)$ cases.

3) Active sensory test: Texture discrimination: Fig. 9 shows the results of the experiment. The horizontal axis indicates the vibration amplitude, and the vertical axis indicates the ratio of correct sandpaper identification. Higher ratios equate to better tactile sensitivity. As the figure indicates, the mean correct ratios for all the controlled cases tended to be higher than that of the no-vibration case. Oneway ANOVA showed a significant main effect from the vibration amplitude $\left(F(5,60)=2.84, p=0.022, \eta_{p}{ }^{2}=\right.$ $0.191)$. The results of post-hoc Dunnett testing also revealed significant differences against the non-vibration case for the $0.5 \mathrm{~T}(p<0.05)$ and $1.0 \mathrm{~T}(p<0.05)$ cases.

4) Motor skill test: Minimal force grasping: Fig. 10 shows the results of the experiment. The horizontal axis indicates the vibration amplitude, and the vertical axis indicates the amplitude of the grasping force as determined by calculating the mean force over a period of 1 second from 1.0 [sec] to 2.0 [sec] in the measured data. Smaller forces equate to better motor performance in terms of pinch grasping. Improvements in motor performance were observed for all the controlled cases. One-way ANOVA showed a significant main effect from the vibration amplitude $\left(F(5,60)=2.95, p=0.018, \eta_{p}{ }^{2}=0.197\right)$. The results of post-hoc Dunnett testing also revealed significant differences against the no-vibration case for the $0.75 \mathrm{~T}$ $(p<0.01)$ and 1.25T $(p<0.05)$ cases.

\section{B. Discussion}

Table I - Table IV show the results of the Dunnett tests for all experiments. The results of the passive sensory tests support the findings of previous studies investigating 




Fig. 10. Results of grasping test: grasping forces for all of the controlled cases were smaller than that of the no-vibration case. Statistically significant differences were observed for $0.5 \mathrm{~T}, 1.0 \mathrm{~T}$, and $1.25 \mathrm{~T}$ ( $* *$ means $p<0.01$ and $*$ means $p<0.05)$.

the effect of SR on the improvement of tactile sensation [14], [15]. These observations also indicate that whitenoise vibration from the radial side of the fingertip has a significant effect in improving tactile sensitivity. The results of the texture discrimination test validate the usefulness of the sensorimotor enhancer for tasks requiring active high-precision sensing capability, such as manual surface finishing. The results of the motor skill test also indicate that the sensorimotor enhancer improves grasping force optimization. The perception of partial slip on the contact surface of the fingertip plays a key role in realizing stable grasp for humans [27], [28]. These results suggest the possibility that the improvement of tactile sensitivity may also improve fingertip slip perception.

Some of the results obtained indicate that vibration from the radial side of the fingertip improves sensorimotor performance even if its amplitude is supra-sensory (1.25T or more). It should be noted that the viscoelasticity of the fingertip attenuates vibration. In a previous study, the

TABLE I

RESULTS OF DUNNETT TEST IN THE TWO-POINT DISCRIMINATION TEST

\begin{tabular}{c|cc}
\hline \hline Dunnett test & $F$ statistics & $p$ value \\
\hline No-vib - 0.5T & 0.47 & 0.6558 \\
No-vib - 0.75T & 3.94 & $\mathbf{0 . 0 0 0 3}$ \\
No-vib - 0.1T & 3.02 & $\mathbf{0 . 0 0 6 7}$ \\
No-vib - 1.25T & 1.69 & 0.1539 \\
No-vib - 1.5T & 0.93 & 0.4450 \\
\hline \hline
\end{tabular}

TABLE II

RESULTS OF DUNNETT TEST IN THE ONE-POINT TOUCH TEST

\begin{tabular}{c|cc}
\hline \hline Dunnett test & $F$ statistics & $p$ value \\
\hline No-vib - 0.5T & 3.61 & $\mathbf{0 . 0 0 1 0}$ \\
No-vib - 0.75T & 4.10 & $\mathbf{0 . 0 0 0 2}$ \\
No-vib - 0.1T & 3.81 & $\mathbf{0 . 0 0 0 5}$ \\
No-vib - 1.25T & 0.75 & 0.5301 \\
No-vib - 1.5T & 0.66 & 0.5729 \\
\hline \hline
\end{tabular}

authors investigated fingertip vibration transmissibility and found that the amplitude becomes progressively smaller and the delay becomes larger for points farther from the position where vibration is applied [24]. Contact-related fingertip deformation also affects vibration transmissibility characteristics. Sophisticated design for the sensorimotor enhancer, including the point at which the actuator is attached, may increase the effect of SR on tactile sensitivity and motor performance.

\section{CONCLUSiOnS}

This paper presented the results of an experiment involving a prototype of a wearable sensorimotor enhancer for fingertip sensitivity. The device was designed to stimulate tactile receptors inside the fingertip using a compact piezoelectric actuator attached to its radial side. This design keeps the palmar region free and helps to maintain the wearer's manipulation ability. Sensory and motor tests were conducted to determine the efficacy of the device, and the results confirmed its potential usefulness.

Although further investigation is necessary to clarify the effects of tactile sensitivity on other types of motor performance, the development of a wearable device for fingertips is expected to assist individuals in workplaces, including those engaging in laboratory work with palpation for tumors, handling of very small objects, texture design for products and high-precision manual assembly. Continued research in this area may also lead to the development of a novel device that helps individuals with incomplete peripheral neuropathy to use their hands more reliably in daily activities and at work.

Several different actuator attachment designs can also be considered. For example, actuators could be attached to both the radial and ulnar sides. In such cases, the effective position at which vibrations from multiple actuators are well synchronized can be determined in consideration of vibration transmissibility characteristics. In addition, an actuator could be mounted on the fingernail so that it can effectively transmit applied vibration in a role similar to

TABLE III

RESULTS OF DUNNETT TEST IN THE SANDPAPER DISCRIMINATION TEST

\begin{tabular}{c|cc}
\hline \hline Dunnett test & $F$ statistics & $p$ value \\
\hline No-vib - 0.5T & 2.79 & $\mathbf{0 . 0 2 8 7}$ \\
No-vib - 0.75T & 1.36 & 0.5170 \\
No-vib - - 0.1T & 2.60 & $\mathbf{0 . 0 4 6 2}$ \\
No-vib - 1.25T & 0.80 & 0.8859 \\
No-vib - 1.5T & 0.00 & 1.0000 \\
\hline \hline
\end{tabular}

TABLE IV

RESULTS OF DUNNETT TEST IN THE MINIMAL FORCE GRASPING TEST

\begin{tabular}{c|cc}
\hline \hline Dunnett test & $F$ statistics & $p$ value \\
\hline No-vib - 0.5T & 2.10 & 0.0704 \\
No-vib - 0.75T & 3.11 & $\mathbf{0 . 0 0 5 1}$ \\
No-vib - 0.1T & 2.19 & 0.0577 \\
No-vib - 1.25T & 2.30 & $\mathbf{0 . 0 4 4 4}$ \\
No-vib - 1.5T & 0.95 & 0.4350 \\
\hline \hline
\end{tabular}


that of a speaker cone. Further investigation of vibration transmissibility is needed for optimal design of the sensorimotor enhancer.

\section{ACKNOWLEDGMENTS}

The authors wish to thank Maxi Hertanto, Danielle Martin, Morgan Hunter, Kazuki Namima, Muhammad Salman, and Dr. Karim Sabra for their support in the experiments.

\section{REFERENCES}

[1] R. Benzi, A. Sutera, and A. Vulpiani. The mechanism of stochastic resonance. Journal of Physics A, 14(11):453-457, 1981.

[2] R. Benzi, G. Parisi, A. Sutera, and A. Vulpiani. Stochastic resonance in climatic change. Tellus, 34(1):10-16, 1982.

[3] B. McNamara and K. Wiesenfeld. Theory of stochastic resonance. Physics Review A, 39(9):4854-4869, 1989.

[4] J. K. Douglass, L. Wilkens, E. Pantazelou, and F. Moss. Noise enhancement of information transfer in crayfish mechanoreceptors by stochastic resonance. Nature London, 365:337-340, 1993.

[5] J. J. Collins, T. T. Imhoff, and P. Grigg. Noise-enhanced information transmission in rat sal cutaneous mechanoreceptors via aperiodic stochastic resonance. Journal of Neurophysiology, 76(1):642-645, 1996.

[6] J. B. Fallon and D. L. Morgan. Fully tuneable stochastic resonance in cutaneous receptors. Journal of Neurophysiology, 94:928-933, 2005.

[7] J. J. Collins, T. T. Imhoff, and P. Grigg. Noise-enhanced tactile sensation. Nature London, 383:770, 1996.

[8] J. J. Collins, T. T. Imhoff, and P. Grigg. Noise-mediated enhancements and decrements in human tactile sensation. Physical Review E, 56(1):923-926, 1997.

[9] E. Simonotto, M. Riani, C. Seife, M. Roberts, J. Twitty, and F. Moss. Visual perception of stochastic resonance. Physics Review Letter, 78:1186-1189, 1997.

[10] F. Zenga, Q. Fub, and R. Morsec. Human hearing enhanced by noise. Brain Research, 869:251-255, 2000.

[11] H. R. Dinse, T. Kalisch, P. Ragert, B. Pleger, P. Schwenkreis, and $M$. Tegenthoff. Improving human haptic performance in normal and impaired human populations through unattended activation-based learning. ACM Transactions on Applied Perception, 2(2):71-88, 2005.

[12] N. T. Dhruv, J. B. Niemi, J. D. Harry, L. A. Lipsitz, and J. J. Collins. Enhancing tactile sensation in older adults with electrical noise stimulation. Neuroreport, 13(5):597-600, 2002.

[13] L. Khaodhiar, J. B. Niemi, R. Earnest, C. Lima, J. D. Harry, and A. Veves. Enhancing sensation in diabetic neuropathic foot with mechanical noise. Diabetes Care, 26(12):3280-3283, 2003.

[14] N. Harada and M. J. Griffin. Factors influencing vibration sense thresholds used to assess occupational exposures to hand transmitted vibration. British Journal of Industrial Medicine, 48:185-192, 1991.

[15] G. A. Gescheider, S. J. Bolanowski, J. V. Pope, and R. T. Verrillo. A four-channel analysis of the tactile sensitivity of the fingertip: frequency selectivity, spatial summation, and temporal summation. Somatosensory \& Motor Research, 19(2):114-124, 2002.

[16] A. Priplata, J. Niemi, M. Salen, J. Harry, L. Lipsitz, and J. Collins. Noise-enhanced human balance control. Physical Review Letter, 89(238101-1-238101-4), 2002.

[17] S. E. Ross, B. L. Arnold, J. T. Blackburn, C. N. Brown, and K. M. Guskiewicz. Enhanced balance associated with coordination training with stochastic resonance stimulation in subjects with functional ankle instability: an experimental trial. Journal of NeuroEngineering and Rehabilitation, 4(1), 2007.

[18] W. Provancher and N. Sylvester. Fingerpad skin stretch increases the perception of virtual friction. IEEE Transactions on Haptics, 2(4):212-222, 2009.

[19] T. Ooka and K. Fujita. Virtual object manipulation system with substitutive display of tangential force and slip by control of vibrotactile phantom sensation. In Proc. of IEEE Haptics Symposium, pages 215-218, 2010.

[20] S. A. Mascaro and H. H. Asada. Measurement of finger posture and three-axis fingertip touch force using fingernail sensors. IEEE Transactions on Robotics and Automation, 20(1):26-35, 2004.
[21] M. Tanaka, K. Miyata, and S. Chonan. A wearable braille sensor system with a post processing. IEEE/ASME Transactions on Mechatronics, 12(4):430-438, 2007.

[22] H. Jeong, M. Higashimori, and M. Kaneko. Improvement of vibration sensitivity by tangential vibration. 21(4):554-562, 2009.

[23] J. M. Romano, S. R. Gray, N. T. Jacobs, and K. J. Kuchenbecker Toward tactilely transparent gloves: Collocated slip sensing and vibrotactile actuation. In Proc. of World Haptics Conference, pages 279-284, 2009.

[24] Y. Kurita, M. Shinohara, and J. Ueda. Wearable sensorimotor enhancer for a fingertip based on stochastic resonance. In IEEE International Conference on Robotics and Automation, pages 3790 3795, 2011.

[25] A. W. Freeman and K. O. Johnson. A model accounting for effects of vibratory amplitude on responses of cutaneous mechanoreceptors in macaque monkey. Journal of Physiolosy, 323:42-64, 1982.

[26] S. J. Bolanowski, G. A. Gescheider, R. T. Verrillo, and C. M. Checkosky. Four channels mediate the mechanical aspects of touch. Journal of the Acoustical Society of America, 84:1680-1694, 1988.

[27] R. S. Johansson and G. Westling. Roles of glabrous skin receptors and sensorimotor memory in automatic control of precision grip when lifting rougher or more slippery objects. Exp. Brain Res., 56:550-564, 1987.

[28] M. Tada and T. Kanade. An imaging system of incipient slip for modelling how human perceives slip of a fingertip. In Proc. of IEEE International Conference on Engineering in Medicine and Biology Society, pages 2045-2048, 2004. 